\title{
Probing General Relativity with Accreting Black Holes
}

\author{
A. C. Fabian \\ Institute of Astronomy, \\ Madingley Road. \\ Cambridge CB3 0HA \\ UK \\ email: acf@ast.cam.ac.uk
}

\begin{abstract}
Most of the X-ray emission from luminous accreting black holes emerges from within 20 gravitational radii. The effective emission radius is several times smaller if the black hole is rapidly spinning. General Relativistic effects can then be very important. Large spacetime curvature causes strong lightbending and large gravitational redshifts. The hard X-ray, powerlaw-emitting corona irradiates the accretion disc generating an X-ray reflection component. Atomic features in the reflection spectrum allow gravitational redshifts to be measured. Time delays between observed variations in the power-law and the reflection spectrum (reverberation) enable the physical scale of the reflecting region to be determined. The relative strength of the reflection and power-law continuum depends on light bending. All of these observed effects enable the immediate environment of the black hole where the effects of General Relativity are on display to be probed and explored.
\end{abstract}

Keywords. Black holes, X-ray astronomy, active galactic nuclei

\section{Introduction}

Black holes are a common feature of the Universe. They have long been suspected to be responsible for the prodigious powers of quasars (Lynden-Bell 1969) and Galactic X-ray binaries such as Cygnus X-1 (Tananbaum et al. 1972). In these cases it is accretion of matter into the black hole which makes these objects luminous. We do not of course "see" the black hole here, but the matter swirling around it in the accretion flow which is heated by the gravitational energy released. This process is the most efficient known, in terms of the fraction of rest mass released, after matter-antimatter annihilation, with a typical value of ten per cent, which is about 20 times higher than hydrogen fusing into helium.

Much of the energy is released in the strong gravity regime very close to the black hole at only a few gravitational radii $\left(r_{\mathrm{g}}=G M / c^{2}\right)$ enabling the effects of general relativity to be probed by observation. Strongly curved spacetime leads to large gravitational redshifts, strong light bending and if the black hole is spinning, dragging of inertial frames, revealed through the Innermost Stable Circular Orbit or ISCO. We concentrate here on luminous accreting black holes and in particular on their X-radiation. The observed rapid variability seen in some Active Galactic Nuclei (AGN) has long shown that the Xray emission originates from a physically small region. Relativistically blurred reflection components and reverberation now show just how small a region this can be. 

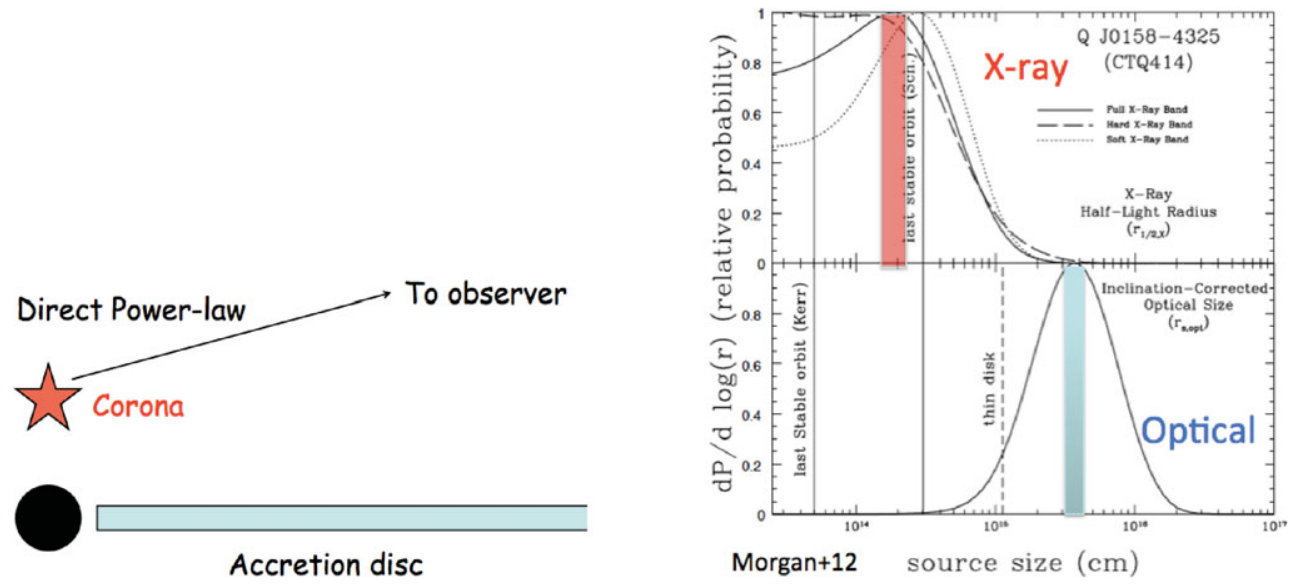

Figure 1. Left: The primary emission components of a luminous accreting black hole consist of the power-law continuum emitting corona and the quasi-black body disc. Right: Estimates of the half-light radii of the X-ray (upper) and optical (lower) emitting regions of the doubly-imaged lensed quasar Q 0158-4325 obtained from optical and X-ray monitoring of its rapid microlensing variability (Morgan et al. 2012).

\section{X-ray Reflection}

Matter accreting onto a black hole is most unlikely to fall in radially but will have sufficient angular momentum to go into orbit about it. Viscosity then causes the matter to spiral inward while the angular momentum is transferred outward. This forms an accretion disc which will be dense, optically thick and physically thin provided that the gravitational energy released is radiated locally. The emitted spectrum is a quasiblackbody of temperature of about $10^{7} \mathrm{~K}$ for a luminous disc around a stellar mass black hole and drops to about $10^{5} \mathrm{~K}$ for a billion solar mass black hole. The accreting gas will therefore be a hot dense plasma with the differential rotation winding up the magnetic fields (which provides the viscosity). In a manner similar to the production of coronal magnetic structures on and above the Sun, we can expect that magnetic structures will occur above the inner accretion disc. Magnetic reconnection in this corona can accelerate particles in the corona and, through inverse Compton scattering of soft disc photons, produce a hard power-law continuum.

Such a configuration (Fig. 1, left) explains the basic spectral components of an Active Galactic Nucleus (AGN); a big blue bump of quasi-blackbody emission from the disc itself and a hard power-law of X-rays extending to hundreds of keV. The coronal power-law emission can be rapidly variable due to its magnetic nature. The rapid X-ray variability seen in many sources shows that the power-law source in most accreting black holes is compact in size. Clear evidence of this compactness is provided by microlensing studies of quasars which are multiply imaged by an intervening galaxy (e.g. Morgan et al. 2012). The half-light radius for the X-ray emission for Q 0158-4325, shown in Fig. 1, is less than $6 r_{\mathrm{g}}$.

The irradiation of the dense disc by the coronal power-law continuum provides a further spectral component, X-ray reflection (Fig. 2). This is just the back-scattered emission plus fluorescence, recombination and bremsstrahlung (see Fabian \& Ross 2011 for a review). It consists of a hard Compton hump together with a soft excess of re-emission including emission lines. The strongest such line is usually iron $\mathrm{K} \alpha$ at $6.4-6.95 \mathrm{keV}$, depending on the ionization state of iron in the disc. It is likely that the irradiation will be intense 


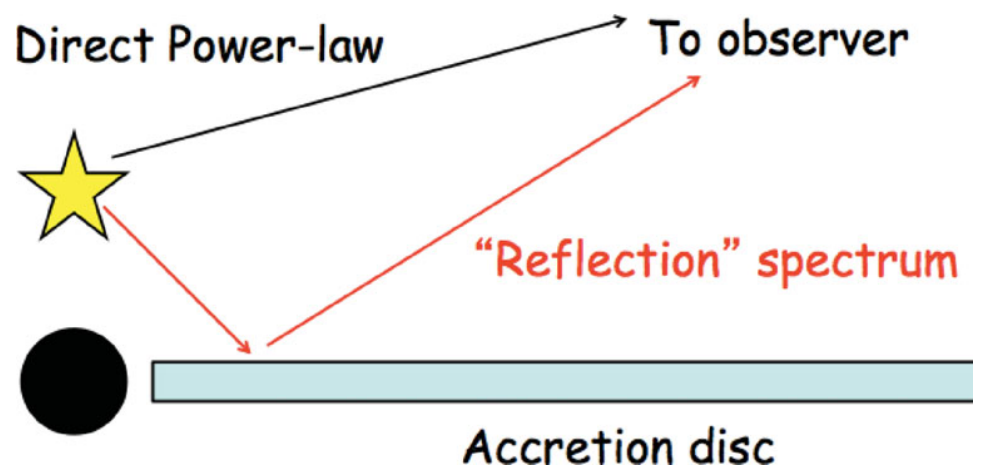

Figure 2. Power-law X-ray source, indicated by the yellow blob representing the corona, irradiating the inner regions of the accretion disc about a black hole. The paths of the primary and reflected components are shown.
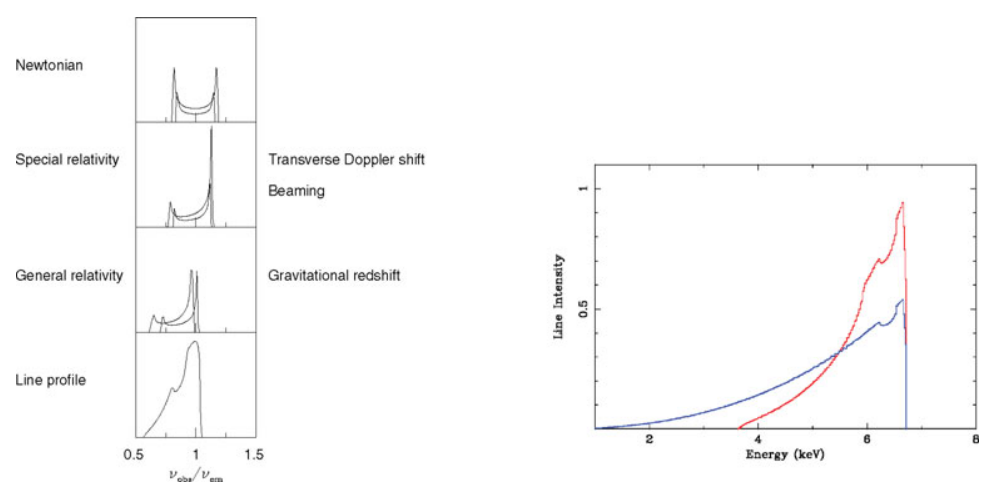

Figure 3. Left: Expected line profiles from 2 radii in an orbiting disc showing Newtonian, Special and General Relativistic effects. The lower panel shows the broad skewed line expected from the whole disc. Right: Broad iron line expected from a non-spinning Schwarzschild black hole (red) and a maximally spinning Kerr black hole (blue).

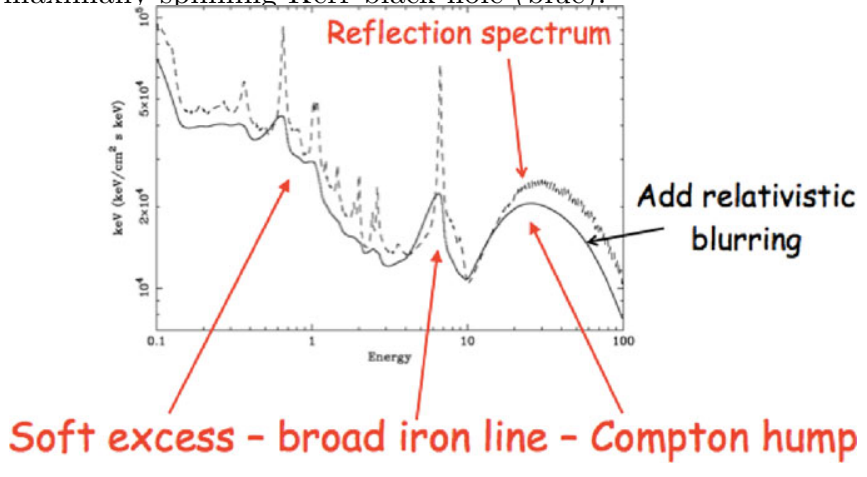

Figure 4. Relativistically-blurred reflection spectrum (unblurred shown by dashes) from Ross \& Fabian (2005).

enough to control and raise the ionization state above that expected from the hot disc alone.

Having emission lines produced from the disc is very important, since if they are observed then we can measure the Doppler shifts and thus the velocity of the accretion flow, which can be up to half the speed of light. We can also measure the gravitational redshift, which can tell us the radius at which the emission originates (Fig. 6, Fabian et al. 1989; Laor 1991). We expect an accretion disc to extend down to the ISCO within 

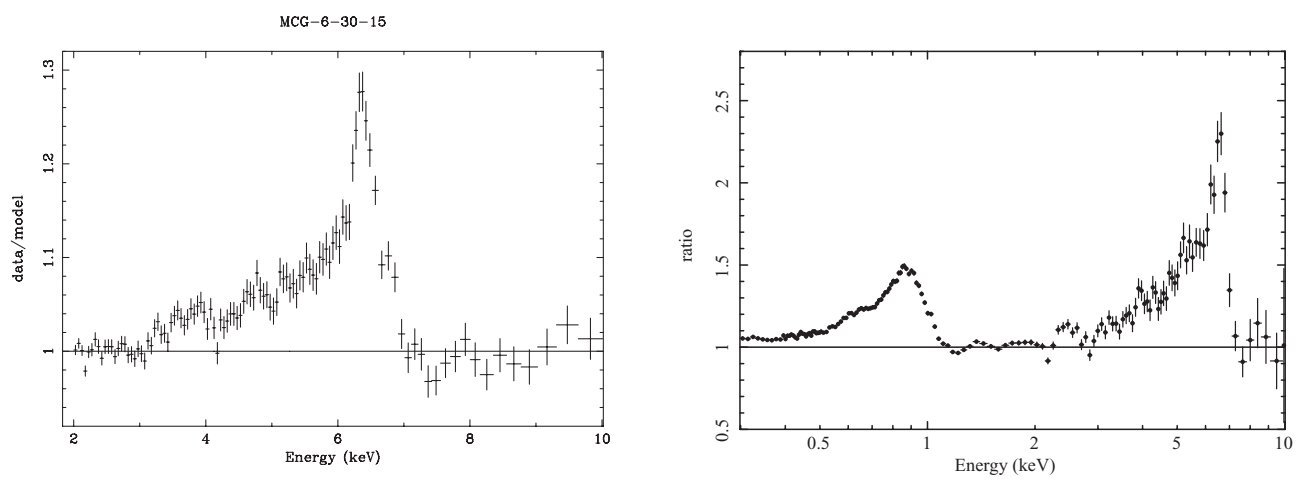

Figure 5. Left: Broad iron-K line in MCG-6-30-15 as seen with Suzaku (Miniutti et al. 2007). The large red wing, extending to lower energies, is principally due to the effects of gravitational redshifts close to the black hole. Right: Broad iron-K and iron-L lines in 1H0707-495 (Fabian et al. 2009). The small bump in the red wing of the iron-L line could be due to an oxygen-K line.
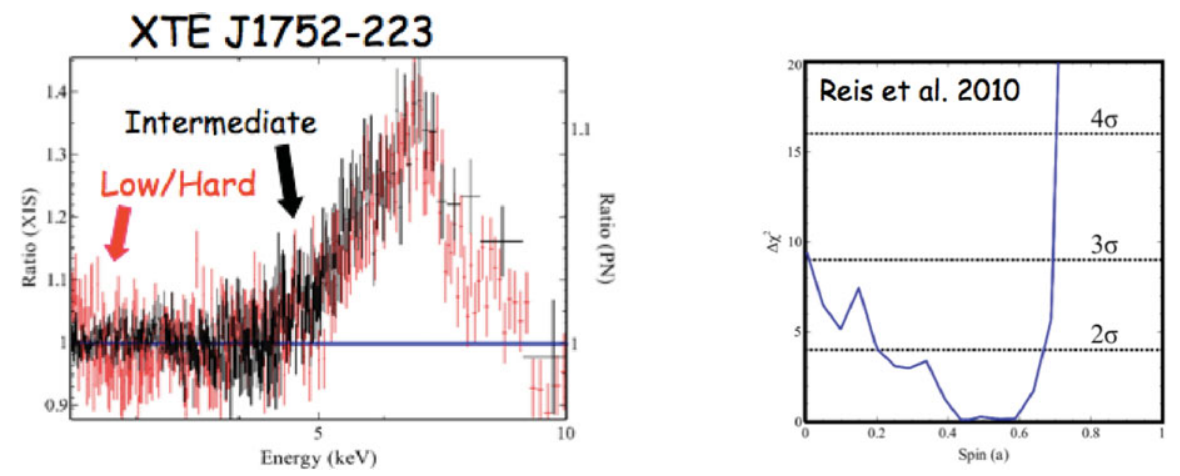

Figure 6. Left: Spectrum of the broad iron line in BHB XTE J1752-223 (Reis et al. 2011). The line profile is unchanged with spectral state. Spectral fitting shows that the black hole spin is about 0.55 .

which the matter plunges on a ballistic orbit into the black hole. Since the radius of the ISCO depends on the spin of the black hole, measurement of the largest gravitational redshift translates to a measurement, or at least a lower limit on, the black hole spin.

The net observed spectrum from the inner parts of an accretion disc around a black hole therefore consists of a power-law continuum, a reflection spectrum blurred by Doppler shifts and gravitational redshifts, a soft excess below $2 \mathrm{keV}$, a broad iron line from 4$7 \mathrm{keV}$ and a Compton hump peaking around $30 \mathrm{keV}$ (Fig. 4). The broad iron line was first seen from an AGN (MCG-6-30-15) with the Japanese-US satellite mission, ASCA (Tanaka et al. 1995). A recent version of the spectrum from this object using Suzaku is shown in Fig. 5 (Miniutti et al. 2007). The line is so broad that indicates a high spin $(a>0.95)$, with the ISCO well within radius $r=2 r_{\mathrm{g}}$, where GR effects must be very strong. Broad iron lines have been found from a range of Seyfert 1 AGN (Nandra et al. 2007, Brenneman \& Reynolds 2009), Galactic Black Hole Binaries (BHB, Miller 2007) and neutron star systems (Cackett et al. 2008). An example from a BHB is shown in Fig. 6 where the broad line of XTE J1752-223 appears not to change between the intermediate and hard state during its 20? outburst (Reis et al 2010). Fitting the spectrum with a reflection model reveals the black hole spin to be about 0.55 .

The discussion has so far ignored absorption and outflows (e.g. fast and slow warm 

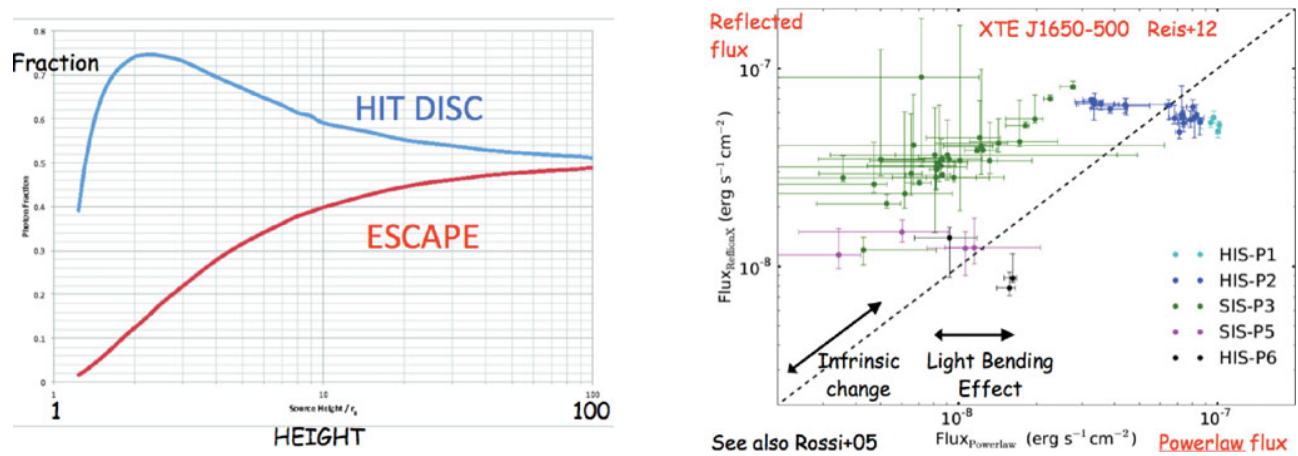

Figure 7. Left: Fraction of photons from an isotropic sources which hit the disc (top blue line) or escape to infinity (lower red line) as a function of source height. Right: Intrinsic power-law flux plotted against flux in the reflection component in the outburst of the Galactic BHB XTE J1650-500 (Reis et al. 2012). Intrinsic source changes cause motion along a diagonal of positive gradient, changes of coronal height cause motion along a diagonal of negative to zero gradient. The different coloured points indicate different states of the source. The evolution of the source in the plot, starting from the upper right, is interpreted as follows: the leftward shift of the points during the High Intermediate State (HIS) is due to the corona dropping in height, it then weakens in intrinsic luminosity during the Soft Intermediate State (SIS) before rising back in height et the end of the SIS.

absorbers) which clearly occur in some luminous accreting black holes. Some (e.g. Miller et al. 2008) argue that a dense outflow completely obscures all emission from within tens to hundreds $r_{\mathrm{g}}$, hides the strong gravity regime from view. The absorber then mimics the relativistically blurred reflection features described above. How such mimicry works for both AGN and BHB is not explained. Many BHB are bright enough that any such extreme obscuration would be obvious. It is far more likely that absorption and outflows are just a complication that can be corrected for in spectral models. Microlensing, rapid X-ray variability and reverberation results all point to the strong gravity regime being directly observable in most Type I AGN.

\section{Light Bending}

The iron line in MCG-6-30-15 is stronger than expected and, although variable, does not show the same level of variability as the power-law continuum (Fabian \& Vaughan 2003). These are likely to be due to strong light bending (Strength: Martocchia \& Matt 1996, 2002; Variability: Miniutti \& Fabian 2004). Strong gravity close to the black hole bends the light towards it, causing it to be focussed on the disc. In so doing it makes an intrisically isotropic continuum source appear anistropic to the outside observer (Fig. 7). Fewer photons escape to infinity and more strike the disc, or fall into the hole as the corona lies closer to the black hole. Good evidence for light bending was noted in the BHB XTE J1650-300 by Rossi et al. (2005). A recent reworking of the data on that source by Reis et al. (2012) shows this result more clearly (Fig. 7).

Further evidence in support of extreme light bending emerged from early in 2011 when colleagues discovered that 1H0707-495 was dramatically reducing in flux and going into a low state (Fig. 8). The soft flux from the source reduced by over an order of magnitude in January and February before recovering in March. We triggered an observation of the object with XMM, under an accepted programme of Norbert Schartel for studying low states in AGN. The spectrum of the source now looked similar in shape to when brighter, although of course much reduced in flux and most interestingly shifted to lower energies. The corona had dropped to within $2 r_{\mathrm{g}}$ of the black hole (Fig. 8). The shape 

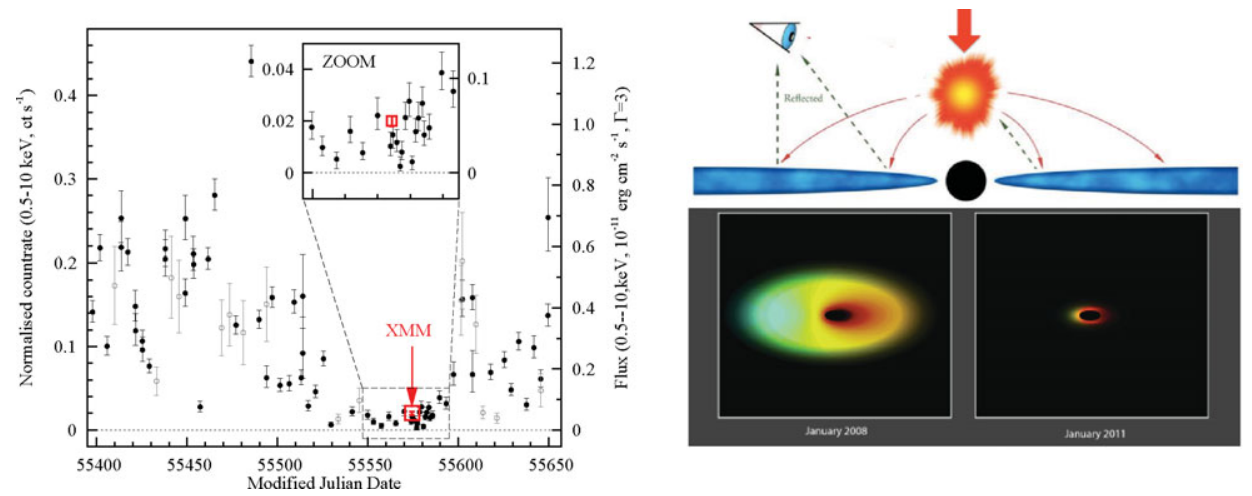

Figure 8. Top: Emissivity profile of 1H 0707-495 in 2008 and 2011 (Fabian et al. 2012). The outer parts of the corona disappeared in early 2011 (bottom panel, courtesy Dan Wilkins). of the emissivity profile of reflection (deduced from the shape of the broad iron line) required the very strong gravitational light bending expected very close to the black hole (Wilkins \& Fabian 2011, 2012a).

Several other AGN have been seen to drop to a reflection-dominated phase, explainable by extreme light bending due to the corona collapsing to the centre (e.g. PG2112, Schartel et al. 2007; Mkn 335, Gallo et al. 2012; PHL 1092, Miniutti et al. 2012).

\section{Reverberation}

Recently, we have seen both the iron K $\alpha$ and L $\alpha$ lines in the AGN 1H 0707-495 (Fig. 5). This object is a very highly variable type of AGN known as a Narrow Line Seyfert 1 galaxy (NLS1). The detection of the L $\alpha$ line is possible here since the abundance of iron is particularly strong. The result is from a very long (500 ks) XMM-Newton exposure which has also enabled the detection of X-ray reverberation for the first time (Fig. 9, Fabian et al. 2009). This means that the reflection-dominated emission below $1 \mathrm{keV}$ lags behind the power-law which dominates the spectrum above $1 \mathrm{keV}$, owing to the difference in light paths taken by the direct power-law and by reflection (Fig. 2). The lag is about $30 \mathrm{~s}$ which corresponds to about $2 r_{\mathrm{g}}$ for a $2 \times 10^{6} \mathrm{M}_{\odot}$ black hole, such as is suspected in $1 \mathrm{H} 0707-495$. The results imply that most of the primary coronal X-ray source is very compact and centred close to the black hole above the inner accretion disc. We have now obtained similar lag results from another NLS1, IRAS13224-3809 (also plotted in Fig. 9; Fabian et al. 2012).

A key test of the reverberation/reflection picture is the energy dependence of the lags. Reflection occurs over a wide range of radii in the disc. Variations in the primary continuum should be rapidly followed by variations in the inner reflection, where the gravitational redshift is strongest, so appearing in the red wing of the broad line. The outer parts of the disc, giving the blue horn of the line, respond slower and give larger lags (Fig. 10 left). This is indeed what is observed in the first reverberation results from the iron-K band obtained in the X-ray bright AGN, NGC 4151 (Fig. 10 right, Zoghbi et al. 2012). Iron $K \alpha$ lags are clearly seen with the most rapid (higher temporal frequency) variations showing shorter lags $(\sim 1000 \mathrm{~s})$ at more redshifted energies $(4-5 \mathrm{keV})$ than the slower (lower temporal frequency) variations showing larger lags $(\sim 2500 \mathrm{~s})$ close to the rest energy of 6-7 keV. Iron $\mathrm{K} \alpha$ lags are now also seen in the in the deepest observations of 1H 0707-495 and IRAS13224-3809 (Kara et al. 2012a,b).

A study of 32 AGN by Barbara De Marco et al. (2012) lists 15 more AGN (9 above a significance of $3 \sigma$ ) showing rapid reverberation in soft X-rays (Fig. 9). The lag timescales 

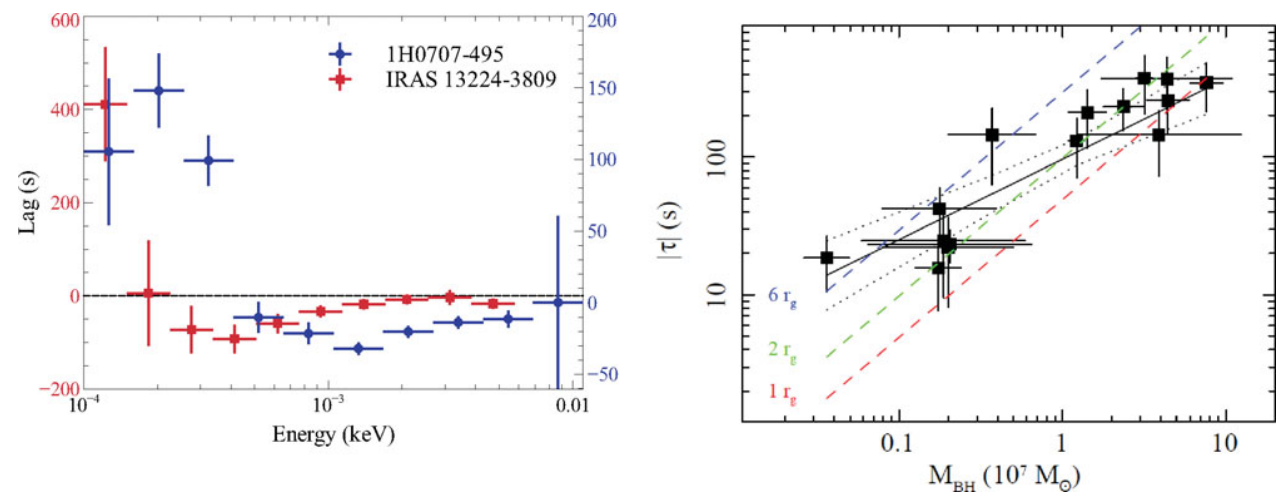

Figure 9. Top: Soft vs. Hard Lags in 1H 0707-495 and IRAS13224-3809 as a function of frequency. At the highest frequencies the lag is negative meaning that changes in the reflectiondominated soft band lags behind those in the power-law-dominated hard band (Fabian et al. 2009; 2012). Right: Timescale of soft lags (15 detected out of 32 sources examined) plotted versus black hole mass (De Marco et al. 2012). The light crossing time of 1,2 and $6 r_{\mathrm{g}}$ are shown by dsahed lines.
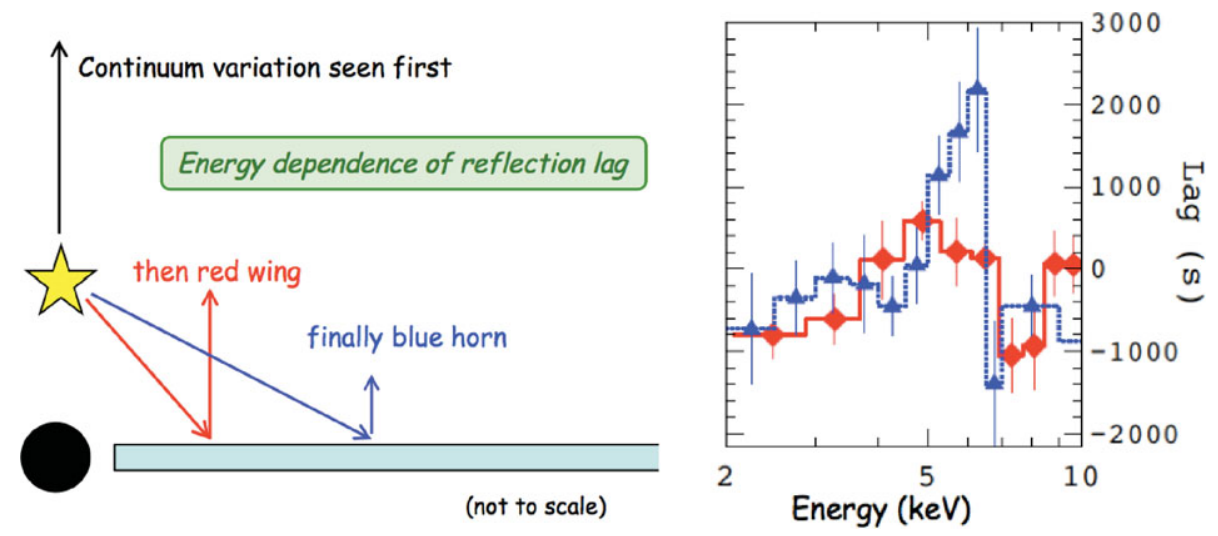

Figure 10. Left: Schematic explanation of the energy dependence expected from reflection lags. A variation in the continuum is seen first then detected in the reflection spectrum from the innermost part of the disc so in the red wing of the iron line, and finally arrives from the outer part of the disc, seen in the blue horn. Right: Lag energy spectra for NGC 4151 (Zoghbi et al. 2012). The shorter, high frequency lags (red) peak at $4-5 \mathrm{keV}$ in the red wing of the lines, originating closest to the black hole, whereas the larger, lower frequency lags (blue) show a narrower spectral peak at 6-7 keV.

correlate with mass and show that most of the reverberation originates from within a few gravitational radii. This naturally leads to the conclusion that soft X-ray emission from many AGN originates close to the ISCO around moderately to highly spinning black holes. Finally, the similarity between the geometry of AGN and BHB is emphasised by the discovery of ms timescale lags in the BHB by Uttley et al. (2011).

Note that there is a bias in any flux-limited sample of AGN towards highly-spinning objects if the distribution of mass accretion rate at large radii is the same for all spins (Brenneman et al. 2011). The mass to radiation conversion efficiency of an accretion disc increases by a factor of 3 or more as the spin is increased.

A detailed discussion of the energy and frequency development of the lags, including the Shapiro delay, is given by Wilkins \& Fabian (2012b). 


\section{Summary}

We now have very good observational evidence of the strong gravity regime around black holes in AGN and BHB. X-ray observations enable us to probe General Relativistic effects such as large gravitational redshifts, strong light bending and an ISCO at radii implying dragging of inertial frames. The overall picture obtained in this way is consistent with GR but does not yet test it. That may come about through testing models in which GR is modified (e.g. Johanssen \& Psaltis 2012) or, possibly, through a combination of more precise light bending (i.e. space) and reverberation (time) measurements.

\section{Acknowledgements}

I am grateful to the Conference Organisers for the opportunity to talk at this interesting meeting. Thanks to my many collaborators, including Dan Wilkins, Erin Kara, Dom Walton, Abdu Zoghbi, Rubens Reis, Phil Uttley, Ed Cackett, Jon Miller, Luigi Gallo, Giovanni Miniutti, Chris Reynolds and Randy Ross, and to George Chartas for Fig. 1 (right).

\section{References}

Brenneman, L. W. \& Reynolds, C. S. 2009, ApJ, 702, 1367

Brenneman, L. W., et al. 2011, ApJ, 736, 103

Cackett, E. M., et al. 2008, ApJ, 674, 415

Chen, B., Dai, X., Kochanek, S., Chartas, G., Blackburne, J. A., \& Kozlowski, S. 2011, ApJ, 740, L34

De Marco, B., et al. 2012, MNRAS, arXiv:1201.0196

Fabian, A. C., Rees, M. J., Stella, L., \& White, N. E. 1989, MNRAS, 238, 729

Fabian, A. C. \& Vaughan, S. 2003, MNRAS, 340, L28

Fabian, A. C., et al. 2009, Nature, 459, 540

Fabian, A. C. \& Ross, R. R. 2010, SScRv, 157, 167

Fabian, A. C., et al. 2012, MNRAS, 419, 116

Gallo, L., et al. 2012, arXiv:1210.0855

Johanssen, T. \& Psaltis, D. 2012, arXiv:1202.6069

Kara, E., Fabian, A. C., Cackett, E. M., Steiner, J., Uttley, P., Wilkins, D. R., \& Zoghbi, A. 2012a, MNRAS in press, arXiv:1210.1465

Kara, E., Fabian, A. C., Cackett, E. M., Miniutti, G., \& Uttley, P. 2012b, MNRAS submitted

Laor, A., et al. 2005, ApJ, 620, 744

Lynden-Bell, D. 1969, Nature, 223, 690

Martocchia, A. \& Matt, G. 1996, MNRAS, 282, L53

Martocchia, A., Matt, G., \& Karas V., 2002, A\&A, 383, L23

Miller, J. M. 2007, ARAA, 45, 441

Miller, L., Turner, T. J., \& Reeves, J. N. 2008, $A \mathscr{E} A$, 483, 437

Miniutti, G. \& Fabian, A. C. 2004, MNRAS, 349, 1435

Miniutti, G, et al. 2007, PASJ, 59, 315

Miniutti, G., Brandt, W. N., Schneider, D. P., Fabian, A. C., Gallo, L. C., \& Boller, T. 2012, MNRAS, 425, 1718

Morgan, C. W., et al. 2012, ApJ, 756, 52

Nandra, K, O’Neill, P. M., George, I. M., \& Reeves, J. N. 2007, MNRAS, 382, 194

Reis, R. C., et al. 2011, MNRAS, 410, 2497

Reis, R. C., Miller, J. M., Reynolds, M. T., Fabian, A. C., Walton, D. J., Cackett, E., \& Steiner, J. F. 2012, arXiv:1208.3277

Ross, R. R. \& Fabian, A. C. 2005, MNRAS, 358, 211

Rossi, S., Homan, J., Miller, J. M., \& Belloni, T. 2005, MNRAS, 360, 763 
Schartel, N., Rodríguez-Pascual, P. M., Santos-Lleó, M., Ballo, L., Clavel, J., Guainazzi, M., Jiménez-Bailón, E., \& Piconcelli, E. 2007, A\& A, 474, 431'

Tanaka, Y., et al. 1995, Nature, 375, 659

Tananbaum, H., Gursky, H., Kellogg, E., Giacconi, R., \& Jones, C. 1972, ApJ, 177, L5

Uttley, P., Wilkinson, T., Cassatella, P., Wilms, J., Pottschmidt, K., Hanke, M., \& Böck, M. 2011, MNRAS, 414, L60

Wilkins, D. R. \& Fabian, A. C. 2011, MNRAS, 414, 1269

Wilkins, D. R. \& Fabian, A. C. 2012a, MNRAS, 424, 1284

Wilkins, D. R. \& Fabian, A. C. 2012b, MNRAS, submitted

Zoghbi, A., Fabian, A. C., Reynolds, C. S., \& Cackett, E,M., 2012, MNRAS, 422, 129

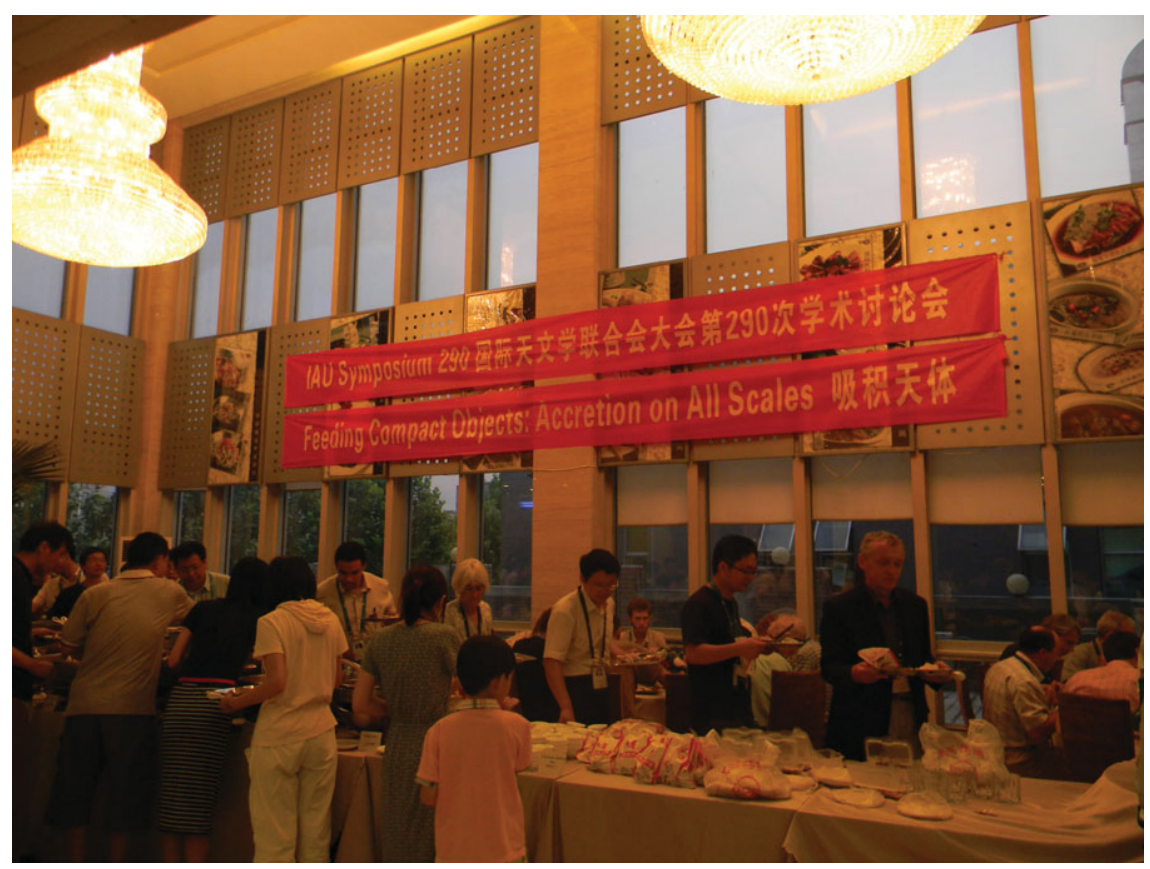


A. C. Fabian
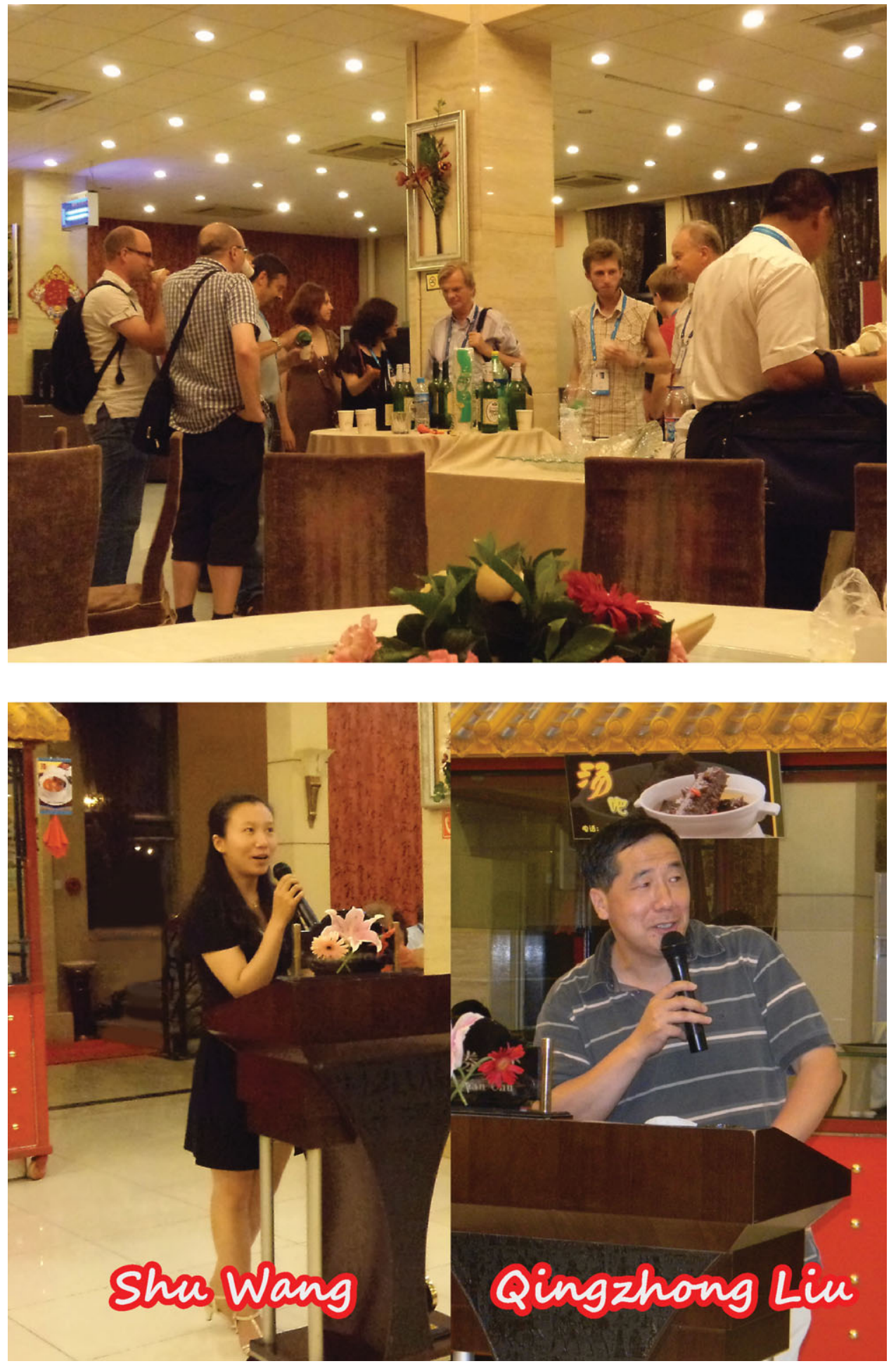